Realidad social, políticas públicas, comunicación y tecnología 


\title{
Realidad social, políticas públicas, comunicación y tecnología; una línea de generación o aplicación del conocimiento dentro del Doctorado en Investigación de la Comunicación
}

\author{
Rafael Tonatiuh Ramírez-Beltrán ${ }^{1}$ \\ Rogelio Del Prado Flores ${ }^{2}$ \\ https://doi.org/10.36105/stx.2018n1.06
}

\section{Resumen}

El presente artículo es un desarrollo de los fundamentos de la línea de investigación y generación del conocimiento (LGAC) Realidad social, políticas públicas, comunicación y tecnología dentro del Doctorado en Investigación de la Comunicación (DEIC). En el documento se trabajan de forma sucinta algunos de los principios teóricos generales, posturas, categorías y esquemas que dan base a esta línea. El artículo explora la definición misma de línea de investigación con diferentes posturas e instituciones. Se da cuenta de los fundamentos, objetivos y principios éticos que se persiguen y de andamios conceptuales iniciales, como base de futuras estructuraciones. Se intenta contextualizar y referenciar el trabajo y definir temáticamente los contenidos a investigar en comunicación. Se documenta el trabajo y procesos de la misma línea y las tareas por venir.

\footnotetext{
1 Profesor de la Facultad de Comunicación e Investigador del Centro de Investigación para la Comunicación Aplicada (CICA) de la Universidad Anáhuac México. Miembro del Colegio de Calidad del Doctorado en Investigación de la Comunicación (PNPC) de la Facultad de Comunicación de la Universidad Anáhuac México. rramirez@anahuac.mx

2 Profesor de la Facultad de Comunicación e investigador del Centro de Investigación para la Comunicación Aplicada (CICA) de la Universidad Anáhuac México. Miembro del Colegio de Calidad del Doctorado en Investigación de la Comunicación (PNPC) de la Facultad de Comunicación de la Universidad Anáhuac México. rogelio.delprado2@anahuac.mx
} 


\section{Palabras clave}

Producción académica, posgrados en comunicación, formación de investigadores en comunicación, desarrollo profesional.

\section{Introducción}

Los posgrados, por una necesidad sustantiva en la generación de conocimientos, están obligados a sostener y encauzar líneas de investigación que sirvan como un núcleo dinámico que articule, colabore en la formación, informe y avance con respecto a objetos de estudio que se problematicen en su interior. Por lo anterior, con el fin de dar marco a la LGAC Realidad social, políticas públicas, comunicación, y tecnología dentro del DEIC de la Universidad Anáhuac México, reconocemos que se podría citar un amplio repertorio de teóricos que han dado cuenta de la transformación sufrida por la sociedad occidental, y en particular en la comunicación, en los últimos 25 años, desde la caída del muro de Berlín, que simboliza el fin de la posibilidad del socialismo real como sociedad alternativa y la permanencia de un capitalismo que se fue deshumanizando en forma dramática en el último cuarto de siglo. De igual forma, el ataque del 11 de septiembre de 2001 a la Torres Gemelas en Nueva York refleja un nuevo escenario geopolítico; las fronteras no son lo que prometen, cada ciudadano del mundo tiene que ser observado, y las telecomunicaciones operan como instrumentos de vigilancia y control frente a la amenaza constante del terrorismo.

Los cambios en la conformación del nuevo escenario geopolítico presentan grandes desafíos para la comprensión de la realidad social. Cada ciudad se convierte en un espacio en el que acontece la globalización. Ésta es estudiada por importantes autores, entre ellos K. Kosík (1963), A. Heller (1977), Berger y Luckmann (1968), Anthony Giddens (19722010), G. Lipovestsky (1983-2015), J. Baudrillard, Noam Chomsky, Naomi Klein, Emanuelle Wallestrein, Z. Bauman (2003-2009), García Canclini (1995), etcétera, todos ellos descriptores y críticos de estos cambios sociales profundos.

En términos de la teoría de la comunicación, recurriremos a trabajos clásicos del campo, como la teoría de las mediaciones (Martín-Barbero, 1987) y de mediaciones múltiples (Orozco, 1991, 1996, 2001), la investigación de la comunicación de masa (Lozano, 1996) y las narrativas transmedia (Gallego Aguilar, 2011; Hidalgo Toledo, 2013). 


\section{Dos esquemas teóricos y referenciales como punto de partida}

Usaremos dos propuestas teóricas en este artículo que nos ayudarán a vertebrar la línea que como un espinazo se amplía con los aportes de los autores antes referidos. Prahalad y Hammel (2007) han establecido en el nuevo contexto planetario grandes tendencias sociales que pueden ir conformando los contornos de la LGCA Realidad social, políticas públicas, comunicación y tecnología (LGAC/RSPPCO) dentro del DEIC. Cada uno de los puntos que citan debe entenderse desde una visión amplia que afecta el contexto social actual, las políticas públicas de la comunicación y la tecnología. Para estos autores, que comenzaron a hablar del capitalismo con rostro social y la importancia de la base de la pirámide social hace más de un lustro, las siguientes se encuentran entre las tendencias más evidentes:

a) La era Post. La que parece irrenunciable y todo menos socialmente justa, la etapa post-industrial, post-moderna, post-deber, hiper-moderna o sobre-moderna capitalista de la globalización (que surge con la caída del muro de Berlín en 1989, pero que se precipitó después del 11 de septiembre de 2001, con los ataques aéreos a Estados Unidos y su consecuente intervención); proceso implacable y sólo en apariencia sutilmente impuesto por el capitalismo que detona en los países haciendo de sus economías nacionales presas de sus demandas y de sus pobladores migrantes (como en el caso de nuestro país, que expulsa medio millón cada año hacia Estados Unidos) y que tampoco, por cierto, toma muy en cuenta los ciclos de recuperación de los recursos naturales. La época post-industrial no significa la renuncia al sector secundario de la economía, sino el movimiento o transformación de las fábricas tradicionales a formas menos reguladas, más tecnologizadas y menos responsables con los trabajadores y consumidores. Sociedades de una alta conectividad tecnológica, que también puede ser nombrada era digital. Con la irrupción de la red de redes y los aparatos inteligentes, la comunicación se aceleró y nos rebasó poniendo a marchar a la humanidad a otro ritmo: el del vértigo de las redes, la comunicación inmediata y la disponibilidad lejana y móvil; las computadoras personales, los celulares inteligentes, las tablet y la obsolescencia tecnológica como destino inmediato y fatal; la irrupción de prosumidores (creadores de contenidos y de agendas públicas) y audiencias. Hecho comunicativo sin precedentes en la historia de la humanidad.

b) La desregulación de todas las actividades productivas y de servicios. No están exentas cuestiones antes controladas por los Estados como la seguridad, la educación, los 
seguros, la publicidad, la salud, el transporte, los medios de comunicación y algunas áreas del medio ambiente, aunque otros autores, como el propio Giddens (2009), sostengan que la época de la desregulación thatcheriana ha llegado a su fin con la crisis económica reciente. Nos tocará ver el desenlace de este nudo, de no menor relevancia en los tiempos por venir: se apretará más la tuerca desregulatoria y neoliberal o se aflojará redefiniendo al Estado y una nueva regulación.

c) El fin de la certidumbre financiera. La claridad de que la única certeza será la volatilidad y la inestabilidad financiera, y que el desarrollo tecnológico es irrefrenable e inalcanzable su materialización y comprensión para la generalidad de los habitantes del planeta. La evidencia de la crisis de 2007-2014 ha puesto a repensar a los gobiernos y ciudadanos informados sobre hasta qué punto el sector financiero puede especular libremente, sin restricción ni controles.

d) Fronteras productivas. Son cada vez más difusos los límites industriales en los que un producto podría tener en diferentes partes de su proceso de elaboración hasta tres continentes y un número indeterminado de mercados. Esto también incluye países como China y cómo se convirtió en la fábrica del mundo; esta condición productiva la hace estar presente en nuestra vida cotidiana y obliga a voltear a ver sus importantes modificaciones recientes en materia ambiental. La comunicación y la tecnología están presentes de muchas formas en esta mutación.

e) Nuevas formas de convivencia. De los procesos de legitimación social que están emergiendo, plasmados en las nuevas normas de convivencia y comunicación social, surgen aquellas que renuncian a la procreación y la familia tal como la conocemos (matrimonio heterosexual e hijos). Controversias sobre las variantes del matrimonio o a cómo la difícil integración de los jóvenes a la vida productiva está modificando la realidad familiar están presentes en México como temas susceptibles de investigarse con las herramientas del campo de la comunicación.

f) La preocupación ambiental. En la investigación de la comunicación existe una gran tendencia a considerar la sustentabilidad como un tema inevitable del presente y del futuro. Como preocupación gubernamental y de la sociedad civil, debida sobre todo a los efectos manifiestos del calentamiento global y los llamados (todavía) fenómenos naturales (huracanes, inundaciones, sequias, temblores, etcétera), presentes de forma inevitable en la agenda públicay que ahora se tratan de resolver con programas de responsabilidad social más que de política pública que los anticipe y amortigüe.

El segundo esquema teórico al que recurrimos en este artículo parte de la teoría de la sobremodernidad y el no-lugar de Marc Augé (1993, 2001, 2015). El autor describe una sociedad 
de modernidad excesiva y desbordada en su transitoriedad. Augé señala tres movimientos: a) el cambio de una sociedad con rasgos de modernidad a una de modernidad saturada: de las familias con un auto, una televisión y un consumo limitado a un cambio de posibilidades de adquisición en apariencia sin límite; b) el movimiento de lo real a lo virtual, ahora con modernísimos dispositivos móviles que conectan al mundo en cualquier lugar y momento o circunstancia, y c) del lugar (familia, hogar, trabajo, escuela, etc.) al espacio de anonimato (carreteras, transportes públicos, salas de espera, etc.), al que define como no-lugar. Hay otros movimientos, tal vez más sutiles, pero igual de determinantes en la sociedad contemporánea: d) de lo formal a lo no formal, desde el empleo hasta ciertas relajaciones sociales; e) de lo humano al dominio tecnológico en lo laboral, lo informativo, el ocio y las relaciones, las formas de contacto, etcétera; f) de lo permanente a lo fugaz, evidente en todas las artes y otras manifestaciones culturales, comunicativas y sociales, y g) de la identidad a las identidades de las regiones, al país; del género al equipo deportivo o partido político; de la raza a los movimientos sociales; de la persona al prosumidor. Cada ser humano carga diversas identidades incluso a lo largo de un día.

También Augé detectaba al principio del milenio tres excesos sociales: de información, de imágenes y de individualismo, hoy tan nítidos que sólo añadiríamos a este último como elemento constitutivo el ser profundamente hedonista.

A estos tres elementos del exceso podemos añadir otros excesos que también influyen en la comunicación de la sociedad actual: excesos de evasión, volatilidad y rapidez, relativismo, consumismo, aburrimiento, necesidad de gratificación, y violencia.

La LGAC/RSPPCO está pensada en este marco social que se transforma todos los días, por lo que investigar en comunicación en esta línea se vuelve una tarea amplia, incluyente y siempre en evolución teórica y con referentes empíricos móviles.

\section{Línea de generación o aplicación del conocimiento: definición y modificaciones}

En este apartado nos concentraremos en ver cómo va definiéndose la línea de generación y aplicación de conocimiento desde los ámbitos institucionales y cómo la están incorporando algunas instituciones de educación superior en forma explícita a sus programas de posgrado.

Para el Programa del Mejoramiento del Profesorado (Promep), una LGAC debe entenderse como (una): 
Serie coherente de proyectos, actividades o estudios que profundizan en el conocimiento como producto de la investigación básica y aplicada con un conjunto de objetivos y metas de carácter académico, en temas disciplinares o multidisciplinares. Es muy frecuente que la generación de conocimiento, en todos los campos, lleve al desarrollo de aplicaciones de tipo innovador en beneficio de la sociedad. (2015).

En la operación, los programas académicos se apropian de este concepto y lo van modificando, y acotando, enfocando o ampliando; por ejemplo, para el Doctorado en Sistemas y Ambientes Educativos de la Universidad Veracruzana, la LGCA es:

En este programa entendemos que una línea de generación y/o aplicación del conocimiento comprende a un grupo de académicos que cultivan una temática común, que desarrollan una intensa vida colegiada (seminarios de estudio, talleres de investigación, gestión colegiada de la docencia), que comparten productos y resultados académicos y son responsables de la formación y seguimiento tutorial de los estudiantes en el marco del programa doctoral. Concebimos que sus integrantes sean doctores de reconocido prestigio y pueden tener un colectivo de colaboradores con diversos grados académicos. Finalmente, consideramos que una línea de generación y/o aplicación del conocimiento se constituye a propuesta de un grupo de académicos que fundamentan su solicitud y que es avalado por el núcleo académico del programa.

La Universidad Autónoma Metropolitana, en su Glosario de términos y definiciones sobre Promep (2005), omite lo de ciencia básica y aplicada, quedando de la siguiente forma:

Serie coherente de proyectos, actividades o estudios con un conjunto de objetivos y metas de carácter académico, en temas disciplinares o multidisciplinares. Es muy frecuente que la generación de conocimiento, en todos los campos, lleve al desarrollo de aplicaciones de tipo innovador en beneficio de la sociedad.

Otras universidades, como la Universidad Autónoma de Nayarit, resaltan la importancia del recorrido profesional y de investigación de los que conforman el núcleo básico de un posgrado como una guía para la generación de las líneas:

Las líneas de generación y aplicación del conocimiento están relacionadas con las necesidades y prioridades de la actividad profesional del programa de posgrado (Conacyt, 2014). Con el fin de definir las Líneas de Generación y Aplicación del Conocimiento (LGAC) congruentes con la orientación y naturaleza del posgrado, se realizó un análisis de los campos temáticos en los cuales confluyen las trayectorias de investigaciones de los profesores que integran el núcleo académico básico del programa. 


\section{Aproximación conceptual LGAC a los objetivos que persigue}

De manera muy general en este marco referencial, contextual e institucional podemos definir LGAC/RSPPCO de la siguiente forma:

La LGAC Realidad social, políticas públicas, comunicación y tecnología se construye a partir de la investigación e indagación permanente, abierta y sistemática en comunicación. Se plantea la pertinencia y vinculación de los investigadores y estudiantes con problemas de la realidad social multideterminada, para transformarla positivamente con ideas, modelos, teorías y productos de investigación que aporten elementos a la construcción de una cultura de la paz, la democracia, la equidad, la sustentabilidad, la comunicación social responsable, la prioridad de la educación y el desarrollo económico, mismos que forman parte de los planteamientos de un buen vivir en sociedad.

Por lo anterior, el trabajo de la LGAC se ha planteado el siguiente propósito general:

Colaborar y consolidar con procesos de formación e investigación, profesionales del campo de la investigación en comunicación, con conocimientos, habilidades, métodos y valores que le permitan llevar a cabo investigaciones originales para la producción de conocimientos nuevos y de alta calidad, fundamentados con base en las metodologías científicas sobre comunicación y la relación de ésta con los procesos económicos, políticos, sociales, tecnológicos y culturales, generación de políticas públicas en comunicación de la sociedad y de las tecnologías de la información y el conocimiento, considerando integrar en la parte de educación de los futuros doctores la capacidad de reflexión propia, crítica-propositiva y que incluye una visión humana, global, ética y responsable. (Rebeil, Arévalo, Ramírez \& Del Prado, 2016, pág. 2).

De este objetivo de la LGAC sobre la comunicación, de la realidad social, las políticas públicas y las tecnologías, desprendemos seis propósitos particulares; a saber:

- Generar conocimiento riguroso con bases científicas y epistémicas de la investigación en comunicación, relacionado con la realidad social, las políticas públicas y el desarrollo tecnológico;

- A partir de un análisis geopolítico, conocer y criticar las diferentes prácticas comunicativas con el fin de comprender la interacción de los diferentes agentes sociales y políticos, y con ello generar conocimiento que permita, de manera reflexiva, justa y con fundamento en la ética, mayores niveles de participación democrática;

- Analizar diversas temáticas de los sistemas, las ciencias de la información, la inte- 
ligencia artificial, las telecomunicaciones y las redes, con el fin de atender, desde una perspectiva ética e interdisciplinaria, y específicamente de la comunicación, las problemáticas sociales, culturales y de transición hacia la democracia del entorno;

- Examinar de manera reflexiva y crítica las diferentes formas de propiedad de las industrias de la comunicación y la tecnología, y cómo ello influye en las audiencias y en el desarrollo local, nacional y global;

- Desarrollar un pensamiento crítico y reflexivo con respecto a las prácticas comunicativas existentes en la sociedad, así como a la influencia que tienen en usuarios y audiencias, con el fin de potenciar la participación social encaminada hacia una mayor democracia;

- Analizar y proponer políticas públicas desde la comunicación, encaminadas a atender y orientar las actividades económicas, políticas, culturales y sociales que aspiren a elevar los niveles de participación en la sociedad con el fin de que éstas sirvan de manera más integral, con base en la ética. (Universidad Anáhuac, 2016)

\section{La fundamentación de la LGAC}

La LGAC de realidad social tiene como fundamento, en primera instancia, las hondas raíces que la filosofía ha desplegado en Occidente desde su simiente clásica (Grecia y Roma), pasando por el pensamiento medieval y renacentista, el iluminismo y la Ilustración y el pensamiento moderno de los últimos siglos del racionalismo y empirismo a la posmodernidad.

La segunda base es la epistemología científica del siglo XIX al momento actual, centrándonos en la constitución de la comunicación como objeto de estudio teórico y sus posibilidades contemporáneas de vinculación con otras disciplinas científicas en una articulación orgánica que posibilite explicaciones complejas de la realidad social comunicativa, la virtualidad y las intervenciones de las políticas públicas respectivas.

La tercera afluente es la aplicabilidad del conocimiento de la línea a los contextos cambiantes de la realidad, la comunicación y los hipermedias del siglo XXI.

Lo anterior hará posible una aproximación a los objetos de conocimiento desde la construcción teórica del conocimiento en comunicación, pero también ontológica, ética, dirigida hacia el bien y la dignidad de la persona; crítica, pero complementaria del pensamiento, la producción y las acciones propositivas que, desde los aportes propios y colegiados, mejoren los procesos de comunicación. 


\section{Marco contextual: Aportaciones a las grandes preocupaciones y problemas nacionales. Abordajes desde el PECIT I}

En la línea de investigación y aplicación estamos convencidos de que no hay trabajo con el objeto que no pase por varios contextos: históricos, teóricos, epistemológicos, metodológico-conceptuales, referenciales, de necesidades reales: empíricas o teóricas.

Para cada investigación, y dependiendo de lo que se indague, se partirá por lo menos de dos diagnósticos sobre cómo se ha construido el campo del objeto: nacionales e internacionales. Esto determinará los discursos, guías y autores dominantes en el campo, así como la producción hegemónica de expertos centrales y periféricos y el estado actual del campo.

Para ello el trabajo de investigación tiene diversos ámbitos y momentos, siempre en una espiral formativa: de gabinete, planteamientos problemáticos, lectura y definición teórico-conceptual, trabajo de definición personal de lo metodológico, seminarios, tutoría, asesoría y opiniones del colegio de calidad, construcción del modelo de investigación, revisiones, modificaciones, trabajo empírico, análisis e inferencias, discusión, conclusiones, propuestas y diseño de políticas o acciones.

Para cada investigación, el proceso formativo puede utilizar muy distintas modalidades:

- Ensayos

- Diálogos

- Trabajo en seminarios

- Consultas

- Participación en eventos académicos externos

- Proyectos académicos

- Agenda de discusiones

- Aplicación de distintas metodologías y técnicas de indagación

- Productos de investigación

- Propuestas que emergen de las investigaciones

- Mecanismos de difusión de resultados.

\section{La investigación en la LGAC es un objeto integrador}

La LGAC recurre a las diversas metodologías de investigación en comunicación, tanto cuantitativas como cualitativas o hermenéuticas, dialécticas e históricas, siempre con una mirada vigilante de la coherencia y pertinencia académica. 
La LGAC se pregunta sistemáticamente para cada objeto ¿qué, cómo, dónde, por qué y con qué se investiga y para qué?

Las respuestas se constituyen por medio de una base soportada en elementos teóricos, escuelas y corrientes de la comunicación, una visión compleja de mundo y argumentos y modelos, métodos cualitativos, cuantitativos, mixtos y diversas técnicas.

En la Figura 1 damos cuenta de los nodos que se pueden entrecruzar en una investigación de la comunicación.

FIGURA 1. MAШA DE TRANSVERSALES

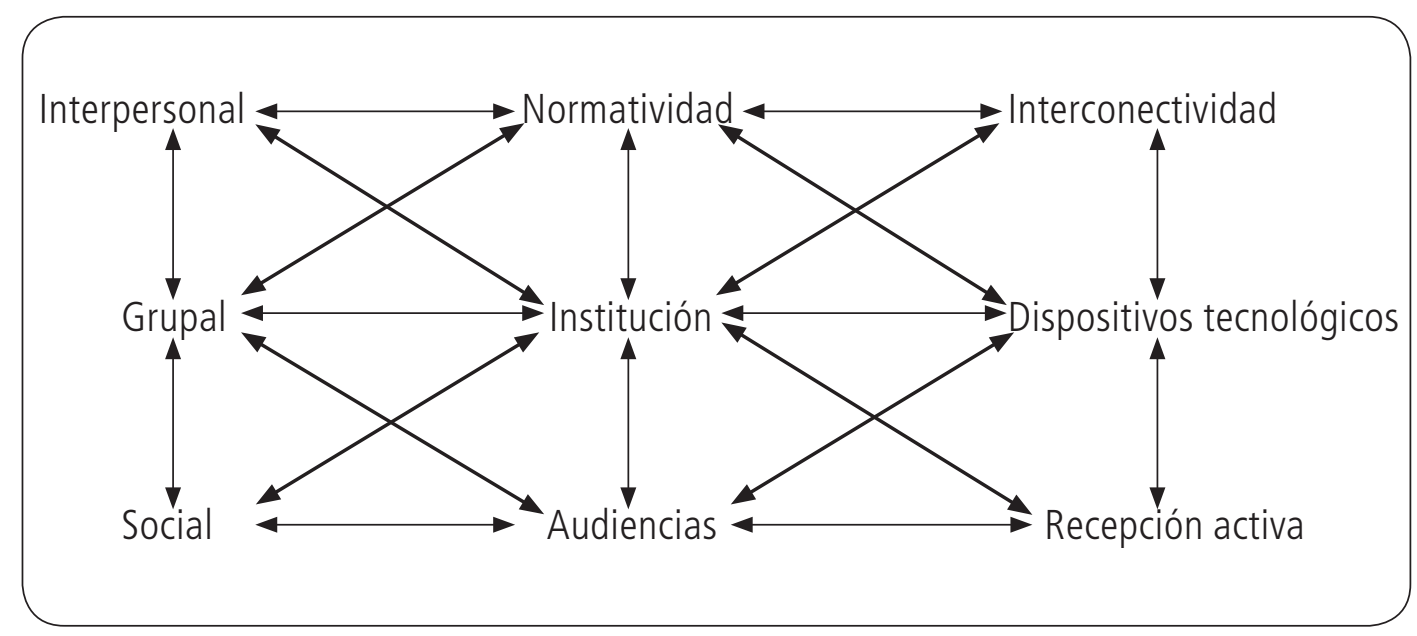

FUENIE: EABORACIÓN PROPIA.

\section{Temas emergentes en la LGAC}

Desde nuestra perspectiva, son muchos los objetos, temas y contenidos que se deberían incluir en esta línea de investigación. La dinámica del programa académico de posgrado nos irá señalando los caminos, trayectos, metas, procedimientos y puertos que tendremos que recorrer. Éstas son sólo unas líneas heurísticas para comenzar a caminar. Sin embargo, y sólo como ejemplo, definimos algunos tópicos susceptibles de investigarse en el futuro inmediato:

- Audiencias, participación social y recepción activa

- El uso de la matemática y la lógica en investigación en comunicación 
- Lo discursivo como método de investigación

- Métodos cualitativos en investigación en comunicación

- Comunicación, política y democracia

- Alfabetización mediática e hipermediática

- Contenidos, plataformas y dispositivos diversos no fijos

- Sociedad del entretenimiento: evasión y trivialización

- Responsabilidad social y comunicación

- La postsociedad de la información y el conocimiento

- Nuevos intereses, límites, formatos y modelos de la publicidad

- Retos de la comunicación y los elementos educativos

- Consumos culturales e industrias culturales postcaída del muro

- Inclusión y elementos y construcción de códigos

- Comunicación y sociedad de la incertidumbre

- Comunicación, inequidad y pobreza

- Comunicación, ambiente y sustentabilidad

- Salud, alimentación y comunicación

- Nuevos retos del periodismo y la comunicación

- Las brechas actuales: económicas, generacionales, raciales, tecnológicas

- Comunicación y migración

- Educación y comunicación.

\section{Reflexiones finales}

El reto que representa el desarrollo de una LGAC dentro de un programa doctoral, en un área como la investigación en comunicación, es mayúsculo. Puede inmovilizar sólo de plantearlo. Sobre todo cuando en el campo de la investigación en esta disciplina se vive con una sensación paradójica: hay grandes cosas ya dichas y argumentadas por investigaciones anteriores que es indispensable revisar y valorar, pero al mismo tiempo nuevas realidades que no invitan a continuar el esfuerzo ya iniciado.

También se vive un razonable vértigo ante una serie de tareas y tópicos que es necesario replantear. Preguntas como las epistemológicas, las metodológicas, las propias del campo, 
o la irrupción de nuevas realidades en comunicación que desbordan los intentos de sistematización nos animan y motivan, más que limitar nuestro interés.

Lo que intentamos en este primer artículo es construir un primer andamio, que pronto será sustituido al ensancharse nuestra mirada y precisarse nuestros focos de atención en la investigación de la comunicación, de los cuales permanentemente daremos cuenta.

\section{Referencias}

Augé, M. (1993). Los no-lugares: espacios del anonimato, antropología sobre modernidad, Barcelona: Gedisa (edición revisada: 2001).

Augé, M. (2015). ¿Qué paso con la confianza en el futuro? México: Siglo XXI.

Bauman, Z. (2003). Comunidad. Argentina: Siglo XXI.

Bauman, Z. (2004). Ética postmoderna. Argentina: Siglo XXI.

Bauman, Z. (2005a). Legisladores e intérpretes. Sobre la modernidad, la postmodernidady los intelectuales. Argentina: Universidad Nacional de Quilmes.

Bauman, Z. (2005b). Modernidad líquida. Argentina/México: Fondo de Cultura Económica.

Bauman, Z. (2005c). Amor líquido. Acerca de la fragilidad de los vínculos bumanos. Argentina: Fondo de Cultura Económica.

Bauman, Z. (2007). Miedo líquido. La sociedad contemporánea y sus temores. Barcelona: Paidós.

Berger, P. L. \& Luckmann, T. (1968). Construcción social de la realidad. Argentina: Amorroutu.

Doctorado en Gestión de las Organizaciones (2 de diciembre de 2015). Universidad Autónoma de Nayarit. Recuperado de http://uae.uan.mx/doctorado-en-gestion-de-organizacion/lineas-de-generacion-y-aplicacion-del-conocimiento-1.

Doctorado en Sistemas y Ambientes Educativos (2 de diciembre de 2015). Universidad Veracruzana. Recuperado de https:/www.uv.mx/veracruz/dsae/docentes-investigadores/lgac/.

Gallego, A. (2011). El diseño de narrativas transmedia. Guía de referencia para las industrias creativas. Colombia: Universidad de Caldas.

García, N. (1995). Consumidoresy ciudadanos. Conflictos multiculturales de la globalización. México: Grijalbo.

Giddens, A. (2009). Recesión, cambio climático y planificación (pp. 25-26). Madrid, España: El País.

Glosario de términos y definiciones sobre Promep conforme a las reglas de operación del Promep 2005 (3 de diciembre de 2015). Universidad Autónoma Metropolitana. Recuperado de http:// coplan.azc.uam.mx/promep/glosarioPromep.htm\#x.

Heller, A. (1977). Sociología de la vida cotidiana. España: Editorial Península.

Hidalgo, J. (2013). Comunicación estratégica y diseño transmedial: tendencias en plataformas móviles. En Congreso Internacional de Comunicación Estratégica CICE 2013, Puebla, Puebla, México. 
Lipovetsky, G. (2005). El crepúsculo del deber: La ética indolora de los nuevos tiempos democráticos. Barcelona, España: Anagrama.

Lipovetsky, G. (2004). El imperio de lo efímero: la moda y su destino en las sociedades modernas. Barcelona, España: Anagrama.

Lipovetsky, G. (2004). El lujo eterno: de la era de lo sagrado al tiempo de las marcas. Barcelona, España: Anagrama.

Lipovetsky, G. (2003). La era del vacio: ensayo sobre el individualismo contemporáneo. Barcelona: Anagrama.

Lipovetsky, G. (2002). La tercera mujer: permanencia y revolución de lo femenino. Barcelona, España: Anagrama.

Lipovetsky, G. (2008). La pantalla global: cultura mediática y cine en la era bipermoderna. Barcelona, España: Anagrama.

Lipovetsky, G. (2006). Los tiempos hipermodernos. Barcelona, España: Anagrama.

Lozano, J. C. (1996). Teoría e investigación de la comunicación de masas. México: Alhambra Mexicana.

Martín, J. (1987). De losmedios a las mediaciones. Comunicación, cultura y begemonía. México: Gustavo Gili. Versión revisada, 1991.

Narrativas transmediáticas (30 de noviembre de 2015). Página principal. Recuperado de http:// www.transmediatico.info/

Orozco, G. (1994). Reflexiones metodológicas sobre la investigación de recepción. En C. Cervantes Barba, Investigar la comunicación. Propuestas iberoamericanas (pp. 171-182). México: Universidad de Guadalajara, Centro de Estudios de Información y la Comunicación, ALAIC.

Orozco, G. (1996). Televisión y audiencias. Un enfoque cualitativo. Madrid: Ediciones De la Torre/ Universidad Iberoamericana.

Orozco, G. (1997). Medios, audiencias y mediaciones. Revista Comunicar (8), 25-30.

Prahalad, C. K. \& Hammel, G. (2007). Competing for the future (p. 384). USA: Harvard Business School Press.

Promep (1 de diciembre de 2015). Cuerpo Académico. En Conceptos básicos. Programa del Mejoramiento del Profesorado. Recuperado de Mover a México: http://promep.sep.gob.mx/ca1/ Conceptos2.html.

Rebeil, M., Arévalo, R., Ramírez, R. \& Del Prado, R. (2016). Línea de generación y/o aplicación del conocimiento. Documento de trabajo para Conacyt. Inédito. México: Universidad Anáhuac/ Centro de Investigación para la Comunicación Aplicada.

Repoll, J. (2010). Arqueología de los estudios culturales de audiencias. D. F., México: Editorial Universidad Autónoma de la Ciudad de México, Colectivo al margen.

Universidad Anáhuac México (2016). Líneas de Generación y/o Aplicación de Conocimiento. En Doctorado en Investigación de la Comunicación. Recuperado de http://pegaso.anahuac.mx/ cica/index.php?option=com_content $\& v i e w=$ article $\& i d=121 \% 3$ Alineas-de-investigacion $\&$ catid $=38 \% 3$ Adoctorado\&Itemid $=205$ 